\title{
Special issue: Universal Thermal Climate Index (UTCI)
}

\author{
Glenn R. McGregor
}

Published online: 14 April 2012

(C) ISB 2012

This special issue represents the outcome of the European COST Action 730 (Cooperation in Science and Technical Development) project which brought together scientists from 19 European countries plus experts from Australia, Canada, Israel and New Zealand to develop a Universal Thermal Climate Index (UTCI). Although a direct output of COST Action 730, the UTCI has its origins in an International Society of Biometeorology Commission of the same name formed in 2002 .

The UTCI, as originally conceived by the ISB Commission, represents a state of the art human thermal climate index in the form of a multi-node thermo-physiological model. It derives its name from the strong desire of the people involved in the ISB Commission and later the COST Action 730 programme, to produce a human thermal climate index that is universal in its utility and application, or in other words is: thermophysiologically significant across the entire range of heat exchange; applicable for whole-body calculations but also for local skin cooling (e.g. frost bite); valid in all climates, seasons, and scales from the micro to macro; is useful for a range of applications in human biometeorology such as daily forecasts and warnings of extreme heat or cold, bioclimatic mapping, urban and regional planning, environmental epidemiology and climate impacts research.

The papers in this special issue present the rational for a "universal" thermal climate index (Jendritzky), describe the nature of the thermo-physiological model which forms the basis of the UTCI (Fiala; Psikuta), assess UTCI uncertainties associated with modeled and observed input values for radiation (Weihs), outline the nature and development of a state of the art adaptive clothing model for the UTCI (Havenith), explain the background to the UTCI operational procedure (Brode), compare the performance of the UTCI with other thermal climate indices (Blazejczyk; Kampmann), present examples of the application of the UTCI to the assessment of thermal climate in outdoor environments (Brode) and address the vexed issue of wind chill (Shitzer).

Besides the UTCI being unique in that it is the first universal thermal climate index, the way in which the UTCI was developed is distinctive. There have been a number of attempts in the past to develop such an index. Such attempts have been frustrated by the fact that "lone researchers" or single discipline teams have tried to solve this problem. This was realised in the early days of the conception of the UTCI with the result that the UTCI has been achieved by a coalition of considerable multidisciplinary expertise with individual scientists going beyond their disciplinary borders to realise the aspiration of a truly universal thermal climate index. The UTCI not only represents a scientific advance but is an exemplar of the co-production of knowledge.
G. R. McGregor $(\bowtie)$

International Society of Biometeorology, School of Environment, University of Auckland,

Private Bag 92019,

Auckland 1042, New Zealand

e-mail: g.mcgregor@auckland.ac.nz 